\title{
A Geographical Proximity Aware Multi-path Routing Mechanism for Resilient Networking
}

\author{
Jinfu Wang, John Bigham, Member, IEEE, and Chris Phillips, Member, IEEE
}

\begin{abstract}
Geographical correlated failures are threats that cause major interruptions and damage to networking systems. To mitigate this rarely addressed challenge, this paper presents a novel geographical location aware route selection algorithm to support uninterrupted networking. The multi-path routing method developed calculates multiple paths that satisfy different constraints while ensuring the prescribed geographical distance metric between selected paths. It is used with existing overlay routing mechanisms to maintain routes. In the evaluation against enhanced k-shortest path algorithms, the new algorithm is shown to provide multi-paths with larger spatial separation and better potential to uninterrupted networking in geographical correlated failures.
\end{abstract}

Index Terms-Routing, Resilience, Communication Networks, Overlay Network, Multipath Routing, Disruption Tolerant, Approximation Algorithm.

\section{INTRODUCTION}

$\mathbf{G}$ EOGRAPHICAL correlated failures can be caused by natural disasters, such as earthquakes [?], tsunami, or by underlying infrastructure problems such as power blackouts [?]. The disruptions caused by correlated failures often have a large geographical span and long duration, and hence can cause severe disruptions to both the underlying communication networks and distributed systems that run on top of them. Mitigation mechanisms against geographical correlated failures have not been studied extensively, despite many responsive overlay routing methods having been designed to address random network degradations or faults. The focus of this paper is to better cope with geographical correlated failures with a novel and effective routing mechanism. A geographical proximity-aware alternative path routing algorithm, which we call GAP, is designed to calculate alternate paths that provide prescribed geographical separation from given primary paths while satisfying other optional constraints. GAP is designed with focuses on medium-sized inter-domain networking over wired substrate network. In this case, for timely delivery and responsiveness requirements, point-to-point overlay paths are preferred over tree-based multicast [?], because scalability is not an overwhelming issue. For example, in a Federated overlay of Message-Oriented Middleware [?], each gateway overlay node may be located in a separate administrative domain. This routing method is shown to provide multi-paths

Jinfu Wang is with Samsung R\&D Institute China, Beijing and National Key Laboratory of Tunable Laser Technology, Harbin Institute of Technology, 100028, China (email: jinfu.wang@samsung.com)

John Bigham (email: john.bigham@qmul.ac.uk) and Chris Phillips (email: chris.i.phillips@qmul.ac.uk) are with the Department of Electronic Engineering \& Computer Science, Queen Mary University of London, E1 4NS, UK

Manuscript received April 2, 2017; revised April 2, 2017. with better geographical separation and better chance providing uninterrupted networking against geographical correlated failures.

\section{BACKGROUND AND RELATED WORK}

The focus of this work is to find alternate paths that are geographically separated to a required extent from the primary path. In the previous work [?] a detailed probability model of earthquake impact is incorporated into the path selection algorithm, rather than the more generalized proximity metric used in GAP. The proximity of networking elements is a key factor determining damage magnitude in many such failure scenarios, e.g., power grid failures, hurricanes, fires and malicious attacks, hence in this work geographical proximity is used to capture the correlation between two geo-locations rather than assuming a specialized probability model.

Constructing a resilient overlay topology [?][?] to mitigate underlay failures and dynamics is a related research field. In this paper the routing algorithm is considered to be independent of the overlay topology, hence the overlay topology is assumed to be in place a priori. The mapping of underlay nodes IP and geolocation can be provided by ISPs and commercial databases. IP-geolocation mapping algorithms have also been researched to estimate unknown locations [?][?]. During recent years, companies like Google and Baidu have continuously improved accuracy of the IP-geolocation information for location based business.

Disjoint path algorithms are not suitable here because in the calculated disjoint path set the primary shortest path of the network may not be included and a complete disjoint path may not always exist in many circumstances. Hence designs that are different from node disjoint routing in wireless ad hoc networks [?] need to be explored. Because finding all possible paths between two nodes is NP-Hard, the most common alternative path selection algorithms are variants of K-shortest path algorithms (KSP). An enhanced KSP algorithm is used to benchmark the performance of GAP. After all paths are sorted an additional path-checking procedure is performed to choose, e.g., two alternative paths that satisfy the desired constraints for acceptable paths, such as geographical separation. A pre-trip partially disjoint alternative route planning approach [?] uses a scheme based on increasing the weight of edges on the primary path $P_{0}$ to search for partially disjoint alternative paths. In contrast our GAP algorithm focuses on finding alternative paths based on geographical separation, not partial disjointedness. Furthermore, GAP uses a self-adjusted penalty based on geographical separation, instead of a fixed 
penalty value for overlapping edges between paths. The GAP algorithm is introduced in the following section

\section{Computing Resilient Proximity-AwARe MuLTipaths}

With the substrate topology and geographical information available, the problem of finding geographical separated alternative paths is described as follows. Given a primary path $P_{0}$, and pre-defined distance threshold $r$ between the primary and alternative paths, the aim is to find an alternative path $P_{a}$ that is as geographical separated as possible with $P_{0}$, whilst also satisfying other constraints such as delay and hop count. Formally the problem is defined as given $G(V, E, W), P_{0}$ and $r$ find $\operatorname{ArgMin}_{P a} P F\left(P_{a}, P_{0}\right)$, where Proximity Factor (PF) is a measurement of geographical proximity between paths defined in Section IV. To find such optimal $P_{a}$ with brute force, the first step is finding all possible paths between source and destination node, then the second step is to pick the path with minimum PF while satisfying other constraints. This problem is NP-Hard.

The pre-defined distance threshold $r$ is a design parameter chosen in accordance with the estimated scope of disruption. Its purpose is to specify what geographical distance is desired for the separation between elements of the primary and alternate paths. In order to apply the GAP algorithm effectively, the chosen value of threshold $r$ should depend on the expected failure diameter. Deploying GAP with a distance threshold $r$ larger than the failure diameter yields better performance. The relationship between the threshold $r$ and different actual failure diameters is demonstrated in the evaluation experiments.

This proximity-aware algorithm searches for alternate paths by extending the edge weight-increment in an incremental method and invoking Dijkstras algorithm. The algorithm uses an edge weight-increment as the heuristic to search for alternate paths that are geographical separated defined by distance threshold $r$. With this chosen heuristic, the weight of an edge $w$ is augmented with a computed penalty $\Delta \mathrm{W}$, before the shortest path algorithm is invoked using the augmented weight $w^{\prime}$, i.e. $w^{\prime}:=w+\Delta \mathrm{W}$.

The algorithm starts with an initial large penalty $\Delta \mathrm{W}=$ $\Delta W_{0}$ on all the edges that are within the distance threshold $r$ to any of the routers or overlay nodes on $P_{0}$. This is to ensure that the algorithm can find the alternate path satisfying the proximity constraint between the newly calculated path $P_{1}$ and the primary path $P_{0}$, if such a solution exists.

If $P_{1}$ does not exist, or it fails to satisfy other pre-defined constraints, then $\Delta \mathrm{W}$ is decreased, and the augmented weights on corresponding edges within distance $r$ from primary path are recalculated. These augmented edges and the unchanged edges form a changed graph. The alternate path search algorithm repeats on this modified graph. The decrease of $\Delta \mathrm{W}$ relaxes the heuristic for geographical separation. This is necessary because if a perfect alternative path does not exist, the algorithm compromises on an alternate path with good quality in terms of proximity and other constraints. In the modified graph after the $\Delta \mathrm{W}$ decrease, every edge within distance $r$ from the primary path has a lighter weight, and thus has a higher probability to be chosen in the alternate path selection algorithm.

The steps of this algorithm are summarized in Fig. ??. The initial value of $\Delta \mathrm{W}$, namely $\Delta W_{0}$, is set to the sum of the weights of all edges in the original undirected graph, i.e. $\Delta \mathrm{W}$ $=\Delta W_{0}=\Sigma W_{i j}$, Here, $i$ and $j$ are the ID of vertex $V_{i}$ and $V_{j}$, and the $w_{i j}$ corresponds to the delay in the overlay edge $E_{i j}$ that connects $V_{i}$ and $V_{j}$. Note that in the algorithm the Function returns the shortest distance from node to any of the underlay or overlay nodes on $P_{0}$.

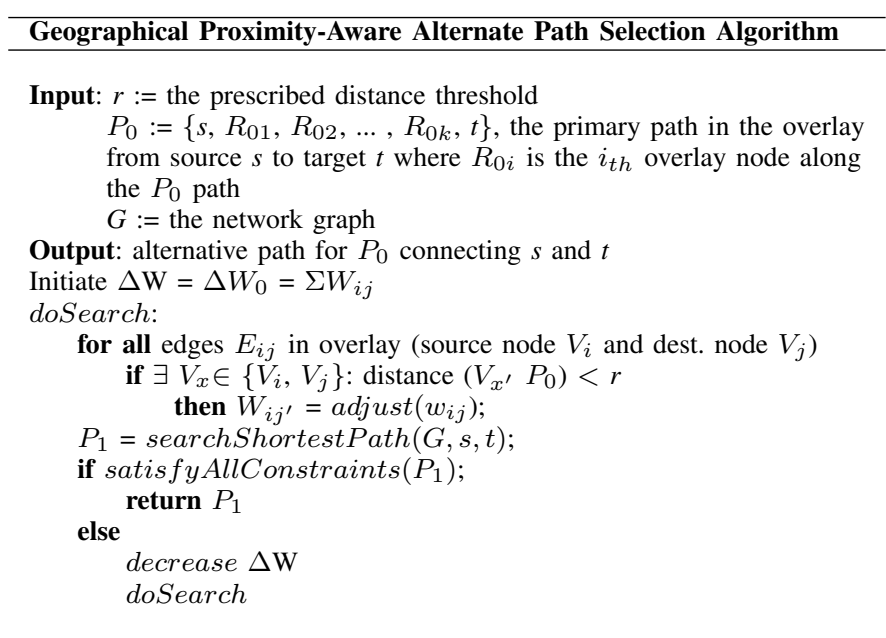

Fig. 1. Pseudo code description of the Geographical proximity-aware Alternate Path (GAP) selection algorithm

Penalty adjustment function $\operatorname{adjust}\left(w_{i j}\right)$ and its variations: The adjustment function $\operatorname{adjust}\left(w_{i j}\right)$ in each loop, returns an augmented weight $w^{\prime}$ by adding a penalty value to the weight of edge $(i, j)$, if $(i, j)$ is within the range threshold: $w_{i j}{ }^{\prime}:=w_{i j}+\Delta \mathrm{W}$, where the initial value of penalty in the first loop is $\Delta \mathrm{W}=\Delta W_{0}$. In a next loop, the penalty is calculated by decreasing its previous value by a coefficient $\alpha(0<\alpha<1)$, i.e. $\Delta \mathrm{W}=\alpha \Delta \mathrm{W}$.

This original adjustment function can be tuned in two respects in order to reduce the total number of loops and to improve the penalty granularity. When attempting to make the algorithm converge in fewer loops, the penalty is decreased logarithmically, instead of by a multiplicative coefficient, i.e. $\Delta W_{n+1}=\log \Delta W_{n}$. Here after the nth iteration, the penalty value for the next iteration is decreased to $\log \Delta W_{n}$. Then to increase the probability of selecting less proximate paths, the penalty added to an $E_{i j}$ within distance threshold $r$ is further adjusted according its distance $d_{i j}$ from the primary path (the more distant paths are given smaller penalty):

$$
\Delta W_{i j}=\left(\left[r-a_{i j}\right] / r\right) \Delta W_{i j}
$$

\section{Evaluation of GAP Multipath Routing}

This part evaluates the performance of GAP against other alternative algorithms in two regards: (1) The geographical separation between paths is evaluated directly by introducing a new metric called the Proximity Factor (PF) between paths, and (2) The number of failures i.e., the simultaneous disconnections in both the primary path and alternative path, is a measurement of resilience of the alternate path algorithm. It 
represents the ability of the network to maintain service in the face of failures.

The number of failures also depends on the topology of the network graph and the failures generated. The PF is a direct measurement of geographical separation. Two variants of GAP were tested. The difference between the variants is in how to adjust penalties in the path-finding process. A penalty function approach is used in the genetic algorithm to handle constraints. One variant uses a decreasing penalty by dividing the penalty by a prescribed number (labelled GAP Div), and the other is based on the logarithm of the penalty from last iteration (labelled GAP Log). For multipath routing and alternative path finding, the k-shortest path algorithms are widely used with different enhancements to enforce constraints. For the evaluations an enhanced k-shortest path algorithm (EKSP) is used as a baseline comparison against the GAP algorithms. The simulation results show the PF of EKSP is lower when compared with GAP. This demonstrates that the GAP algorith$\mathrm{m}$ is pushing the alternate paths geographically further from the primary path than with EKSP.

\section{A. Proximity Factor}

A geographical proximity measure is used as a backup path selection heuristic and represents a degree of geographical correlation between two paths. The proximity needs to be modelled so that the quality of the solutions from different algorithms can be evaluated. The measure can also be used by the path selection algorithms as a heuristic.

The Proximity Factor (PF) between an alternative path $P_{n}$ and its primary path $P_{m}$ is defined in the following description as depicted in Fig. ??: If the distance between any pair of nodes along two overlay paths is below the prescribed distance threshold $T_{\text {distance }}$, then the PF is increased by one. In the experiments below the prescribed distance threshold $T_{\text {distance }}$ holds the same value as the GAP distance threshold r, i.e. $T_{\text {distance }}=R_{f}$. Before returning a value, the PF is normalized by the number of hops in the primary path $P_{m}$, hence a smaller $\mathrm{PF}$ is better.

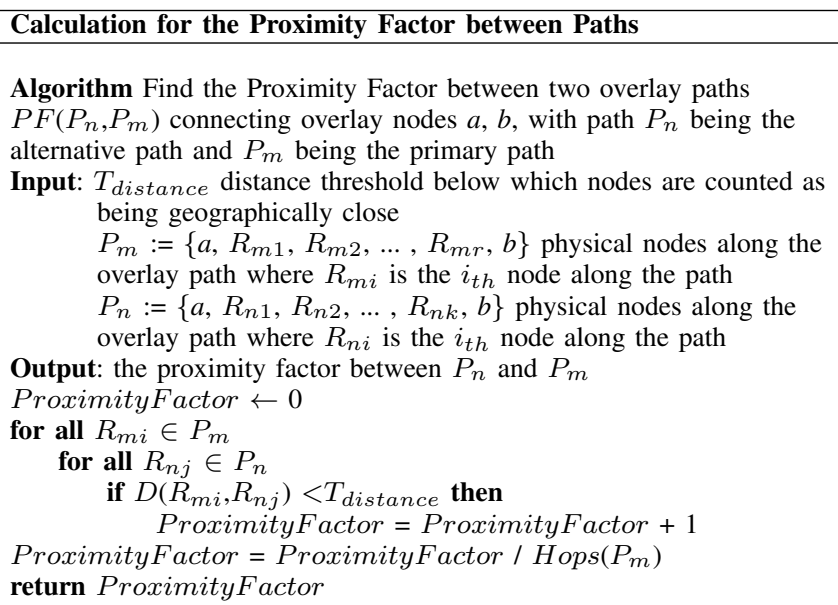

Fig. 2. Pseudo code to calculate the Proximity Factor between paths

\section{B. Benchmark Algorithms}

An enhanced K-shortest path algorithm (EKSP) is implemented for the purpose of comparison with the proximityaware alternate path finding algorithm. KSP calculates and ranks a predefined number of shortest paths according to various preferences. After finding the $\mathrm{K}$ shortest paths between overlay node $s$ and $t$, to find the desired alternate path, an additional path-filtering process is carried out to compare the geographical separation of candidate paths in terms of proximity factor as calculated in Fig. 2 and the path that is the most spatially distant (smallest proximity factor) to the primary path is chosen as the alternative path. In Fig.3, on a $4 \times 4$ network, given the same primary path from node $4 \mathrm{~b}$ to $1 \mathrm{c}$, different alternative paths are found by a brute force algorithm, GAP and EKSP $(\mathrm{k}=5)$. Here $T_{\text {distance }}=1$ and the weight in the graph equals Euclidean distance. In this case, the alternative path of GAP is consistent with the optimal brute force algorithm, and provides better geographical separation than the result of EKSP.

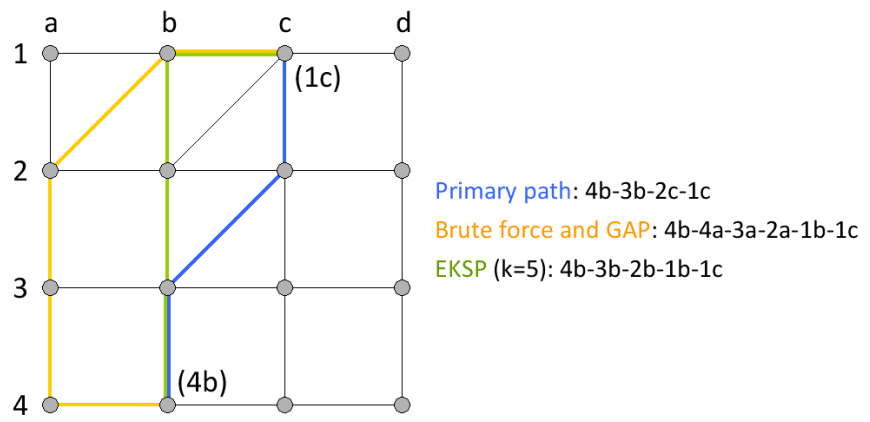

Fig. 3. The primary path and the alternative paths found by brute force, GAP and EKSP algorithms between node $4 \mathrm{~b}$ and $1 \mathrm{c}$

\section{Evaluation of GAP against EKSP}

Correlated failures are rare and difficult to capture in real world testbed such as PlanetLab [?]. Hence we conduct evaluation through simulations. To evaluate the GAP and EKSP algorithms, the underlay graph in the test is generated using the BRITE internet topology generation tool [?]. The graph represents an underlay network comprising 1,000 underlay nodes within a 10,000 by 10,000 Euclidean plane. For the overlay network, 100 overlay brokers are randomly generated, the coordinates of which are drawn from a uniform distribution. Then they are attached to the nearest underlay nodes. 200 overlay source and target node pairs are randomly selected. Between every pair of source and target node, a primary path is established by Dijkstras algorithm, and then the alternative paths are found with the four algorithms.

After the algorithms have found the alternate path solutions, the centres of 50 circular failure regions are randomly generated using a uniform distribution. Each set of 50 failure regions has a predefined failure radius of $R_{f}$, to explore the impact of different failure radius values. The primary and alternate path pairs are evaluated against these failures. The tests are repeated for different values of $R_{f}$. 


\section{Evaluation Results}

After above tests, two sets of statistics are compared in the results. The first is the Proximity Factor, which measures the degree of spatial separation of alternative path algorithms. The second is the number of failures caused by a number of random generated failures. This evaluates the resilience of alternative path algorithms against geographical correlated failures. These are shown in the results in Figure 4 and 5, respectively.

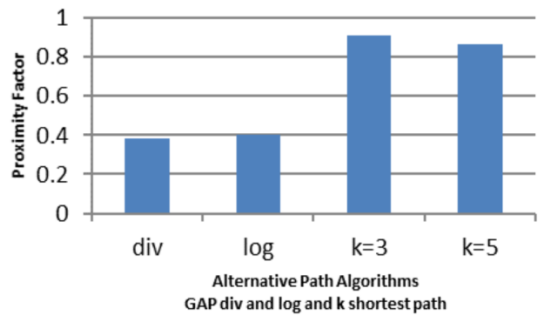

Fig. 4. Proximity Factor between Alternative Paths and Primary Paths (A low PF indicates greater separation)

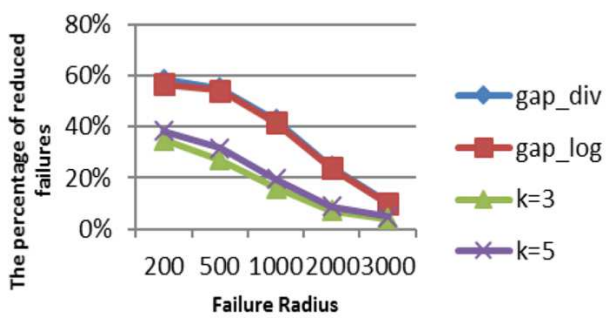

Fig. 5. The percentage of reduced failures of GAP and EKSP compared with using a single primary path. The proportional reduction is defined as: Failures(Primary Path) Failures (GAP or EKSP) / Failures(Primary Path)

In comparison with the EKSP algorithm, the above results in Fig. 4 show that new alternative path algorithm has over a 0.4 decrease in the proximity factor on average. It demonstrates that the new algorithm is pushing the alternate paths geographically further apart from primary paths. As expected, greater geographical separation results in a lower overall number of failures as shown in Fig. 5. The failures are presented as the proportionate reduction in failures relative to using only primary paths.

In the above results, for scenarios of different failure radius, multi-path routing based on GAP and EKSP are compared with a simple shortest overlay path. Both GAP and EKSP based multi-path routing provide improvements over using only a shortest primary path. These improvements are more significant when the failure radius is relatively small. The improvements decrease as the failure radius becomes larger. The proximity constraint threshold $P_{t h}=T_{\text {distance }}=r$ is set to 2000 in all cases. It is shown that for GAP to be most effective, the constraint threshold should be larger than the expected failure diameter. For the smallest failure radius $R_{f}=200$, the improvements are over $58 \%$ and $56 \%$ for GAP approaches and over $34 \%$ and $38 \%$ for EKSP. When the failure radius $R_{f}$ is comparable with $P_{t h},\left(R_{f}=1000\right)$, the GAP approaches maintain a $42.7 \%$ and $41.6 \%$ improvement, while the improvement is significant less for EKSP approaches, being $16.1 \%$ and $19.6 \%$. As the failure radius $R_{f}$, rises above proximity constraint threshold $P_{t h}$, the resilience of the two variants of multi-path routing are notably worse. When the failure radius, $R_{f}$, reaches 3000 , the improvements are $10.2 \%$ and $9.75 \%$ for GAP algorithms and $3.98 \%$ and $4.7 \%$ for KSP-based algorithms. Overall in these tests, both geoaware algorithms improve the resilience of shortest path based routing. However, the GAP algorithms perform better than the EKSP-based ones in all cases.

\section{CONCLUSION}

We presented a novel overlay routing mechanism that is designed to improve the resilience of messaging in response to large scale geographical failures. As a practical improvement to single path overlay routing, this algorithm is developed to search for a geographically diverse alternative path, with any given primary path. It is evaluated against an enhanced kshortest path algorithm with random generated geographical correlated failures. The test network is chosen to provide a suitable geographical representation to effectively evaluate the algorithms and also maintain the average node degree to be representative of realistic networks. In comparison with the EKSP algorithm, for the degree of separation, the novel GAP algorithm improves the degree of spatial separation measured by proximity factor with given primary paths. It demonstrates that GAP is effective in finding alternative paths with appropriate geographical separation. As a result the novel algorithm also successfully increases the percentage of uninterrupted messaging connections in the face of geographically correlated failures.

\section{REFERENCES}

[1] Wang, M. and T. Takada, Macrospatial correlation model of seismic ground motions. Earthquake spectra, 2005. 21(4): p. 1137-1156.

[2] Nedic, D.P., et al., Criticality in a cascading failure blackout model. International Journal of Electrical Power and Energy Systems, 2006. 28(9): p. 627-633.

[3] Esposito, C., D. Cotroneo, and A. Gokhale, Reliable publish/subscribe middleware for time-sensitive internet-scale applications. in Proceedings of the Third ACM International Conference on Distributed Event-Based Systems. 2009, ACM: Nashville, Tennessee. p. 1-12.

[4] Jayalath, C., J. Stephen, and P. Eugster, Universal Cross-Cloud Communication. Cloud Computing. IEEE Transactions on, 2014. 2(2): p. 103-116.

[5] Meersman, R., et al., Overlay Routing under Geographically Correlated Failures in Distributed Event-Based Systems. in On the Move to Meaningful Internet Systems, OTM 2010. Springer. p. 764-784.

[6] Brockmeyer, J.S.a.M., QoSMap: Achieving Quality and Resilience through Overlay Construction. 4th International Conference on Internet and Web Applications and Services, 2009. p. 58-67.

[7] Xian Zhang, Chris Phillips, Physical-aware topology construction and importance of underlying topological information in provider-independent overlays. 6th International ICST Conference on Communications and Networking in China (CHINACOM). 2011. p. 742-746.

[8] Katz-Bassett, E., et al., Towards IP geolocation using delay and topology measurements. in Proceedings of the 6th ACM SIGCOMM conf. on Internet measurement. 2006. p.71-84.

[9] Eriksson, B., et al., A learning-based approach for IP geolocation. in Proceedings of the 11th international conference on Passive and active measurement. 2010, Springer-Verlag: Zurich, Switzerland. p. 171-180.

[10] Su, S.L., et al. A novel design of node-disjoint multi-path QoS routing in multi-hop ad hoc networks. IEEE/IFIP 41st Int. Conf. on Dependable Systems and Networks Workshops (DSN-W). 2011. p. 292-296.

[11] Rouphail, N.M., et al. A decision support system for dynamic pre-trip route planning. in Applications of Advanced Technologies in Transportation Engineering. 1995. p. 325-329.

[12] Spring, N., et al., Using PlanetLab for network research: myths, realities, and best practices. ACM SIGOPS OS Review, 40(1), 2006. p. 17-24.

[13] Alberto Medina, A.L., Ibrahim Matta, John Byers, BRITE: Universal Topology Generation from a User's Perspective. 2001. 